$\frac{7}{1628}$ 
ERNST MARQUARDT

\section{GESCHICHTE WÜRTTEMBERGS}

J. B. METZLERSCHE

VERLAGSBUCHHANDLUNG STUTTGART MCMLXI 
ISBN 978-3-476-99163-8

ISBN 978-3-476-99162-1 (eBook)

DOI 10.1007/978-3-476-99162-1

(C) 1961 Springer-Verlag GmbH Deutschland Ursprünglich erschienen bei J.B. Metzlersche Verlagsbuchhandlung und Carl Ernst Poeschel Verlag GmbH Stuttgart 1961 


\section{VORWORT}

Diese Darstellung der württembergischen Geschichte möchte das politische Schicksal eines süddeutschen Kleinstaates inmitten der großen deutschen und europäischen Mächte in Umrissen erzäblen. Sie bemübt sich, überall den neuesten Stand der wissenschaftlichen Forschung zu geben, will selbst aber nicht als Forschung im eigentlichen Sinn des Wortes gelten.

Wer immer sich mit der württembergischen Geschichte beschäftigt, wird bald auf eine beherrschende Tatsache ibrer Entwicklung stoßen, nämlich auf den Gegensatz zwischen dem Herrscherbaus und den Ständen. Das ständische Wesen ist eine Besonderbeit des Landes, die Württemberg einen eigenen Rang in der deutschen Verfassungsgeschichte anweist. Die Regierungsgewalt lag in der Hand der Landesherren; so eifersüchtig die altwürttembergischen Stände über ibr Steuerbewilligungsrecht wachten, politischer Verantwortung gingen sie meist aus dem Wege.

Die beutige Geschichtsauffassung räumt zwar Fragen der Staatsverfassung und des Staatsrechts einen sebr weitgebenden Einflu $\beta$ auf die Gesamtentwicklung der Völker ein, aber diese Forschung stebt noch am Anfang eines langen Weges und ibr Ergebnis läßt sich nicht abseben. Das ausgezeichnete, für die Landesgeschichte sebr aufscblußreiche Werk von Walter Grube "Fünfbundert Fabre Stuttgarter Landtag 1457-1957" (1957) läßt den Bruch zwischen der alten und der neuen Verfassung Württembergs klar erkennen. Diese stebt im Zeichen der Demokratie des neun- 
zebnten und zwanzigsten Fabrbunderts, jene war bis zu ibrem Ende I805 eine reine Oligarchie, ausgeübt von evangelischen Kirchenmännern und den Vertretern der privilegierten Familien der Ebrbarkeit. Der beiden gemeinsame Name \Stuttgarter Landtagı verdeckt den Gegensatz zweier grundsätzlich verschiedener verfassungsrechtlicher Tatbestände.

In einer politischen Geschichte Württembergs kann die Entwicklung seines kulturellen, wissenschaftlichen und künstlerischen Lebens nur gestreift werden. Um aber den Leser auf das Erbe geistigen Reichtums unserer Heimat wenigstens hinzuweisen, konnte eine von Herrn cand. phil. Ernst Conrad in Tübingen ausgearbeitete und gütigerweise zur Verfügung gestellte synoptische Ubersicht dem Buch beigefügt werden. Die Liste der Regenten Württembergs stellte Herr Dr. Alfons Urble in Tübingen zusammen. Verfasser und Verlag danken vielmals beiden Herren für ibre Mübe und ibre freundliche Hilfe.

Den Herren Professor Dr. Hansmartin Decker-Hauff und Dr. Rudolf Seigel vom Institut für geschichtliche Landeskunde an der Universität Tübingen und Professor Dr. Heinz Gollwitzer, Direktor des historischen Seminars an der Universität Münster/Westf., bin ich für vielfältigen sachkundigen Rat und jede mögliche Förderung zu Dank verpflichtet, den auch hier auszusprechen mir am Herzen liegt.

Herbst 1960

ERNSt MARQUARDT 
INHALT

Vorwort

\section{Erstes Kapitel Seite $1-8$}

SCHWABEN UNTER DEN SÄCHSISCHEN, SALISCHEN UND STAUFISCHEN KAISERN Die Sueben - Deutsche Stämme - Zusammenfassende und auseinanderstrebende Kräfte - Das Lehen - Herzöge von Schwaben - Kloster Hirsau - Klosterprivilegien - Das Haus Hohenstaufen - Reichsfriedensordnung - Staufer gegen Welfen - Kaiser und Papst - Romzüge Schwaben als Hausmacht der Staufer

\section{Zweites Kapitel Seite 9-26}

Die ersten Grafen von Württemberg

Untergang derStaufer, Aufstieg der Württemberger - UlRICH I. ( $\dagger_{1}$ 265) smit dem Daumen - Gründung von Stuttgart - Rudolf von Habsburg, Deutscher König, sein Versuch einer Wiedererrichtung des Herzogtums Schwaben - EberHaRd I. ( $\left.f_{\text {r 325 }}\right)$ >der Erlauchte $<$ - Belagerung von Stuttgart - Der Sühnevertrag vom ro. November I 286 - Reichskrieg gegen Württemberg - Würdigung des Grafen - ULRICH III. († I 344) ein Mehrer des Hausvermögens - Eberhard II. (I315-1392) >der

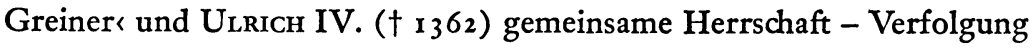
der Juden und Konfiskation ihres Vermögens - Württemberger als Anwärter auf die Königswürde - Der Ebersteinhandel - Streit mit den Städtern: Waffenentscheidungen von Altheim-Reutlingen-Döffingen Eberhard III. (1364-1417) >der Mildes - Die Rittergesellschaft der >Schlegler - Fünf Generationen: Leistung und Ergebnis 


\section{Drittes Kapitel Seite 27-37}

Schwabens Handel und Gewerbe am Ende des Mittelalters Die wirtschaftliche Bedeutung der schwäbischen Reichsstädte - Voraussetzungen des Fernhandels - Der Kreditbrief - Juden und Kawerschen Verkehrsstraßen in Württemberg - Zölle und Geleitbriefe - Handelsgesellschaften - Die Häuser Fugger und Welser, Humpissgesellschaft in Ravensburg - Trust, Syndikat und Monopol - Münzverschlechterung durch das spanische Silber - Krise durch Strukturwandel - Herbst des Mittelalters

\section{Viertes Kapitel Seite 38-66}

\section{Vom TerritorialstaAt zUm Reichsfürstentum}

Eberhard IV. (1388-1419) und Henriette von Mömpelgard - Die Vermögensliste des Hauses Württemberg - Das Böhmische Lehen - Die gemeinsame Herrschaft der Grafen Ludwig ( 141 2-1450) und UlRICH V. (1413-I480) >des Vielgeliebten - Mechthild von der Pfalz - Die Teilung der Grafschaft Württemberg: Uracher und Stuttgarter Linie Die Vormundschaftsregierung - Pfalzgraf Friedrich - Die Pfälzer FehdeDie Niederlage von Seckenheim - Graf Heinrich von Mömpelgard Eberhard V. (1445-1496) im Bart « - Pilgerfahrt nach Jerusalem Fehden - Barbara von Gonzaga - Gründung der Universität Tübingen Verwaltung, Heerwesen, Rechtsprechung - Staat und Kirche - Romfahrt - Finanzen, Steuern, Stände - Wiedervereinigung durch den Münsinger Vertrag - Schwäbischer Bund - Kaiser Maximilian - Reichstag in Worms 1495 - Herzog Eberhard I.

\section{Fünftes Kapitel Seite 67-100}

\section{STURM ÜBER WÜRTTEMBERG}

Eberhard II. (1496-I498) - Willkür und Ausschweifung - Absetzung des Herzogs durch die Stände - Schweizer Krieg - Herzog Ulrich (1487-1550) - Volljährig mit siebzehn Jahren - Erbfolgestreit im Hause Bayern - Austritt aus dem Schwäbischen Bund - Politik im Zickzack >Armer Konrad ` - Die Ehrbarkeit - Konrad Breuning - Tübinger Vertrag - Hans von Huttens Ende - Herzogin Sabina - Hochverratsprozeß, Ambrosius Volland - Kaiserwahl 1519 - Württemberg im Spiel der europäischen Großmächte, Maximilian van Zevenbergen - Vertrieben aus seinem Herzogtum - Versuch der Wiedergewinnung der Herrschaft 
I 524 - Ulrichs Charakter - Württemberg unter österreichischer Hoheit Jahre der Verbannung - Landgraf Philipp von Hessen - Schlacht bei Lauffen a. N. - Kaadener Vertrag - Evangelische Landeskirche - Karl V.Schmalkaldischer Bund - Heilbronn Weihnachten I 546 - Der Felonieprozeß - Interim

\section{Sechstes Kapitel Seite 10I-124}

Des Vaters unähnlicher SohN

Herzog Christoph (1515-1568) - Jugendjahre - Christoph und seine Mutter - Vor den Bundesräten in Augsburg - In Frankreich - Graf Georg - Ein schweres Erbe - Diplomat zwischen Kaiser Karl und König Ferdinand - Passauer Vertrag - Ein Fazit - Reformen in Kirche und Staat - Landesordnung 1 552, Große Kirchenordnung 1559, Förderung von Handwerk und Gewerbe - Schirmherrschaft über die Reichsstadt Eßlingen - Der Herzog und seine Stände - Wahrer seines Rechts Auswärtige Beziehungen - Ausgleich durch friedliche Verhandlung Das Augsburger Bekenntnis - Herzog Ludwig (1554-1593) - Bauherr und Kunstfreund - Getreidegroßhandel der herzoglichen Verwaltung Der Abt von Maulbronn über Zwangswirtschaft und Höchstpreise Vordringen des ständischen Wesens

\section{Siebtes Kapitel Seite 125-142}

\section{LANDSTÄNDE GEGEN FÜRSTENMACHT}

Graf Friedrich von Württemberg-Mömpelgard (1557-1608) - Regierender Graf ${ }_{1581}$ - Unterstützt die Sache der Hugenotten - Absolutist und Merkantilist - Friedrich Herzog von Württemberg $1593-Z$ - Wei Regenten in Württemberg-Huldigung der Stände-Matthäus EnzlinPolitischer Protestantismus unter Friedrichs Leitung - Merkantilismus, Uracher Leinwand - Gründung von Freudenstadt - Konflikt mit den Ständen - JohanN Friedrich (1582-1628) - Prozesse gegen Enzlin und Eßlinger - Union und Liga - Schlacht am Weißen Berg - Herzog Magnus - „Kriegsunternehmer“ - Hexenprozesse - Der fürstbrüderliche Vergleich, die Linien Mömpelgard und Weiltingen

\section{Achtes Kapitel Seite 143-163}

Das LAND IN SEINER ÄRgSTEN Not

EberhaRd III. (I614-1674) - Barbara Sophia von Brandenburg, >Ober- 
vormünderin « - Herzog-Administratoren - Das kaiserliche Restitutionsedikt von 1629 - Jakob Löffler, mömpelgardischer Kanzler - Der Kirschenkrieg - Gustav Adolf von Schweden - Württemberg zwischen Kaiser und König - Eberhard III. 1633 volljährig - Der Tag von Nördlingen - In Straßburg - Kampf für das angestammte Land Friedenskongreß in Münster und Osnabrück, Friedensexekution in Nürnberg - Andreas Burkard, Johann Konrad Varnbüler - Kriegswunden vernarben - Idyll in Héricourt - WiLhelm Ludwig (1647-1677)

\section{Neuntes Kapitel Seite I64-196}

\section{WÜRTTEMBERGISCHES BAROCK}

>Mit-Ober-Vormünderin Land 1677-1692 - Gymnasium Illustre - „Lieber Türken als Reformierte" - Franzosen im Land - Tapfere Frauen - Treffen von Oetisheim Eberhard Ludwig (1676-1733) I693 volljährig - Reform der Reichskreise Franken und Schwaben, Ludwig Wilhelm von Baden und Johann Georg Kulpis - Eberhard Ludwigs militärische Laufbahn - Die Frau Landhofmeisterin Gräfin Würben geb. von Grävenitz - Merkantilistische Wirtschaftspolitik - Schloß Ludwigsburg - Entlastungszeugen: Johannes Osiander und G. B. Bilfinger - KarL AleXander (1684-1737) - Im kaiserlichen Dienst - Glaubenswechsel - Statthalter in Serbien - Regierender Herzog - Die Geschäfte des Hoffaktors Josef Süß Oppenheimer - Umsturzpläne und Legenden

\section{Zebntes Kapitel Seite 197-222 \\ Despotismus und Aufklärung}

KARL Eugen ( $1728-1793$ ) - Der Herzog im Urteil seiner Zeitgenossen Verheißungsvolle Anfänge - Despotische Neigungen - Werkzeuge fürstlicher Willkür: Rieger und Montmartin - Soldatenspielerei - Landschaftskonsulent J. J. Moser - Der Erbvergleich - Ein Geburtstagserlaß Württembergs Hohe Schulen - Revolution im Schlafsaal - Regimentsmedicus Friedrich Schiller - Hof- und Theaterdichter C. F. D. Schubart Ludwig Eugen (I731-1795) - Gutgemeinte Reformversuche - Fortschritt und Reaktion in der Landschaft - Ein Landschaftskonsulent als herzoglicher Diplomat - Friedrich Eugen (1732-I 797) - Württembergischer Fürstensohn in preußischen Diensten 


\section{Elftes Kapitel Seite 223-27I}

\section{REVOLUTION IN WÜRTTEMBERG}

Umsturz von oben - Kämpfe am Rhein und im Schwarzwald - Herzog und Landschaft auf getrennten Wegen - Wechselnde Fronten - Altes Recht und Neue Zeit - Länderschacher beim Rastatter Kongreß FrIedrich II. (1754-1816) - In Rußland - Ehetragödie - Kaltgestellt Schreibtischarbeit eines Frondeurs - Ubernahme der Regierung - Johann Karl von Zeppelin - Ruhe zwischen Herzog und Landschaft - Wechselndes Kriegsglück - Landflüchtig - Im Unglück ungebeugt - Reichsdeputations-Hauptschluß - Kurfürstentum Württemberg - Kampf mit der Landschaft - I805 - Aufhebung der Stände - KöNIG Friedrich Rheinbund - Befreiungskrieg - Wiener Kongreß - Verfassungskämpfe Alt- und Neuwürttemberg, politisch und kulturell

\section{Zwölftes Kapitel Seite 272-307}

Konstitutionelle Monarchie

Freiheit und Einheit - Metternich - Verfassungskämpfe im Stuttgarter Landtag - KöNIG WiLHELM I. (1782-1864) - Treitschke über die „Zaunkönige" - Triasidee - Friedrich List - Liberalismus in Württemberg Das Jahr 48 - Frankfurter Nationalversammlung - Rumpfparlament Auswärtige Politik, Schleswig-Holsteinsche Frage, Krimkrieg - Bismarck in Stuttgart - Zwei-Kaiser-Zusammenkunft - Umrißzeichnung des Königs - Verwaltung und Verfassung des Königreichs

\section{Dreizebntes Kapitel Seite 308-333}

\section{EINGESCHRÄNKTE SOUVERÄNITÄT}

Entstehung der Parteien - Zollverein - Der Volkspartei großer Sieg Karl Mayer - Der Südbund - KöNIG KARL (1823-1891) - Großfürstin Olga von Rußland - Ein neuer Kurs, Karl von Varnbüler Vae Victis! - Königgrätz und Tauberbischofsheim - Ministerwechsel, Hermann Mittnacht - Großdeutsche Politik - Vive l'empereur! - casus foederis - Auf dem Weg zur Einigung - Die Minister Mittnacht und Suckow in Versailles - Jubel über das Reich - Des Hauses Württemberg großes Opfer - Diktat oder Verständigung? - Vernunftehe KöNIG Wilhelm II. (I848-1921) 


\section{Vierzebntes Kapitel Seite 334-346}

\section{STREIFZUG DURCH DIE WIRTSCHAFTSGESCHICHTE}

Vom Agrarland zum Industriegebiet - Wegbereiter - Unternehmer Die Calwer Zeughandels-Kompagnie - Die Engelsaitweberei - Unternehmergeist in Calw - Weinausfuhr und Salzeinfuhr - Der König als Förderer des Wirtschaftslebens - Geheimnisvoller Stahlguß - Württembergische Wertarbeit - Freihandel und Schutzzoll - Langsam, aber sicher!

\section{Fünfzebntes Kapitel Seite 347-358 \\ Ende der Monarchie}

Bundesstaatliche Existenz - Landtagswahl von I 895 - Verfassungsreform, Volkskammer - Große Politik, von Stuttgart aus gesehen Weizsäckers "Württembergische Erinnerungen «: Vorkriegsjahre, Agadir, Verständigung mit England, Einkreisung, die Schüsse von Sarajewo, Innerer Zusammenhang, Rollenverteilung, U-Bootkrieg, Kanzlerkrise Württemberger in der Reichsregierung-Umsturz im Land-Wilhelm II. als König und als Mensch

\section{Sechzehntes Kapitel Seite 359-385}

WÜrtTEMbergische Betrachtungen zUR ZeItgeschichte

Generäle in der Politik - Friede von Versailles und innere Unruhen Kapp-Putsch - Reichsregierung und Nationalversammlung in Stuttgart Württemberg als friedliche Insel - Die neue Verfassung - Landeskirchliche Ordnung - Rechtsregierung - Das Pendel schwingt zurück Wirtschaftskrise - Wendung zum Radikalismus - Nationalsozialismus Machtergreifung - Rückblick auf die wirtschaftliche Entwicklung Schwäche des Weimarer Staates - Adolf Hitler und der deutsche Einheitsstaat - Widerstandsbewegung - Wirtschaftswunder und Atomzeitalter - Ausblick

Stammtafel der Regenten Württembergs (vorderes Vorsatzpapier)

ZeITTAFEL DER EUROPÄISCHEN UND WÜRTTEMBERGISCHEN GESCHICHTE (auf farbigem Papier) Seite $\mathrm{I}^{*}-15^{*}$

Register Seite 386 


\section{ZEITTAFEL}

EUROPÄISCHE

GESCHICHTE

Kaiser Gallienus (260-268) schlägt die Alemannen bei Mailand zurück

Aufgabe des obergermanischen und rhaetischen Limes

482-5 I I Chlodwig

Ubertritt Chlodwigs zum Christentum (soll in der Alemannenschlacht den Ubertritt gelobt haben)

493-526 Theoderich der Große
POLITISCHE

ENTWICKLUNG IN WÜRTTEMBERG

um 260 Landnahme der Alemannen

um 500 Sieg der Franken unter Chlodwig über die Alemannen. Die nördliche Hälfte des Gebietes der Alemannen wird dem Frankenreich einverleibt

Schutzherrschaft Theoderichs über das restliche Alemannien

im Laufe des 7. Jhdts. zunehmender fränkischer Einfluß
GEISTIGES LEBEN IN WÜRTTEMBERG

Aus der Frühzeit der alemannischen Siedlung ist sehr wenig bekannt, da so gut wie gar keine Bodenfunde (nur Funde röm. Münzen)

Beginn des langsamen Prozesses der Christianisierung der Alemannen Erste alemannische Rechtsaufzeichnung, der sogenannte pactus alamannorum Alemannische Gesetzessammlung:

lex alamannorum 
EUROPÄISCHE

GESCHICHTE

7 14-74I Karl Martell;

die Karolinger verdrängen die Merowinger

768-8 14 Karl der Große

in der zweiten Hälfte des 9. Jhdts. Zerfall des Karolingerreiches

919-1024 die sächsischen Könige und Kaiser

919-936 Heinrich I.

936-973 Otto der Große

955 Sieg über die Ungarn auf dem Lechfeld

962 Kaiserkrönung in Rom

973-983 Otto II.

983-1003 Otto III.

1024-II25 die fränkischen oder salischen Kaiser ro24-1039 Konrad II. I033 Burgund kommt ans Reich
POLITISCHE

ENTWICKLUNG IN

WÜRTTEMBERG

746 Einmarsch Karlmanns in Alemannien. Bei Cannstatt (sog. Blutbad von Cannstatt) wird ein Teil der versammelten Großen Alemanniens von den Franken umgebracht. Alemannien wird ein Teil des Frankenreiches. Ende des älteren Stammesherzogtums

nach 900 Entstehung des jüngeren Stammesherzogtums. 918 setzt sich der Hunfridinger Burkhard als Herzog in Schwaben durch (918-926)

Anerkennung von Burkhards Herzogtum durch Heinrich I., Bestimmung seiner beiden nächsten Nachfolger Hermanns I. (926-948) und Liudolfs (948-954), nicht nach einem Erbrecht, sondern durch Eingreifen des Königs, aber Einheirat beider in die alte Herzogsfamilie

973-982 Herzog Otto von Schwaben, dank seiner Freundschaft mit Otto II. 976 auch noch Herzog von Bayern

Herzog Hermann II. (997 bis 1003) tritt nach dem Tod Ottos III. als Thronanwärter auf

Herzog Ernst II. (IOI 5 bis ro3o) erhebt Ansprüche auf Burgund. Er verliert Herzogtum und Leben im Aufstand gegen Konrad II.
GEISTIGES LEBEN

IN WÜRTTEMBERG
Das Kloster St. Gallen und die Klöster auf der Reichenau Mittelpunkte der Bildung der Zeit

Vorstrittsrecht der Schwaben in der Schlacht (der Uberlieferung nach seit der Zeit Karls des Großen; urkundlich sicher spätestens seit dem I 2. Jhdt.). Dadurch Stärkung des Selbst- und Eigenbewußtseins des Stammes

Teilnahme von Schwaben an der Missionierung und $\mathrm{Be}-$ siedlung Böhmens 
EUROPÄISCHE

GESCHICHTE

1056-III6 Heinrich IV. 1073-1085 Papst GregorVII.

Gegensatz Welfen-Staufer I $138-1254$ Könige und Kaiser aus dem Haus der Staufer

I 152-II90 Friedrich I. Barbarossa

I 80 Sturz Heinrichs des

Löwen

I 190-II97 Heinrich VI.

I 2 I 2-I 250 Friedrich II.

1256-1273 Interregnum in Deutschland
POLITISCHE

ENTWICKLUNG IN

WÜRTTEMBERG

Herzog Rudolf I057 bis 1079/80 als Gegenkönig Heinrichs IV. im Kampf gegen denselben

die Staufer Herzöge von Schwaben:

Friedrich I. 1079-1 105, Schwiegersohn Heinrichs IV.

I 268 Konradin, der letzte Herzog von Schwaben und der letzte Staufer wird in Neapel enthauptet
GEISTIGES LEBEN

IN WÜRTTEMBERG

Unter Abt Wilhelm, einem eifrigen Parteigänger des Papsttums im Investiturstreit, wird Hirsau ein Mittelpunkt der von Cluny ausgehenden Reformbewegung. Auch in Schwaben in dieser Zeit Gründung zahlreicher neuer Klöster. - Unter dem Einfluß der Reformbewegung tritt die Pflege der Wissenschaften in den Klöstern stark zurück

Der romanische Baustil entsteht und erreicht seine Vollendung.

Blüte der ritterlichen Dichtkunst. Minnesang:

Friedrich von Hausen,

Reinmar der Alte, Hartmann von der Aue, Gottfried von Straßburg

Aufblühen der Städte; Gründung zahlreicher neuer Städte
Die ersten Württemberger: Konrad (urkundlich erwähnt 1079-1092) gehört im Investiturstreit zur päpstlichen Partei

Seine Enkel Ludwig und Emicho Parteigänger der Staufer. Ludwig erscheint um I1 40 als Graf

1246 Ưbergang der Württemberger zur antistaufischen Partei. Der Untergang des staufischen Hauses begünstigt den raschen Aufstieg der Württemberger
Konrad der vermutliche Erbauer der StammburgWürttemberg im Neckartal bei Cannstatt

Aus Schwaben stammt der Philosoph Albert von Bollstädt (I 193-I 280), als Dominikanermönch Albertus Magnus 
EUROPÄISCHE GESCHICHTE
POLITISCHE

ENTWICKLUNG IN WÜRTTEMBERG

Eigentlicher Beginn der württembergischen $\mathrm{Ge}$ schichte mit Ulrich mit dem Daumen (der Stifter) I 24 I bis 1265
GEISTIGES LEBEN IN WÜRTTEMBERG
1273-1291 Rudolf I. (Graf von Habsburg)

1308-1313 Heinrich VII. (aus dem Haus Luxemburg)

1314 Doppelwahl: 1314-1347 Ludwig der Bayer 1314-1330 Friedrich von Osterreich
I 347-1 378 Karl IV. (aus dem Haus Luxemburg) 1348 Gründung der Universität Prag

1356 Goldene Bulle (Reichsgrundgesetz): 7 Kurfürsten (3 geistliche, 4 weltliche); Bestimmungen über den Landfrieden; den Städten wird die Bildung besonderer Städtebündnisse und die Aufnahme von "Pfahlbürgern" (die außerhalb der Stadt ansässig sind) untersagt
Ihm folgen seine Söhne:

Graf Ulrich II. 1265-1279 und Graf Eberhard der Erlauchte I 279-1325

Kampf mit Rudolf von Habsburg. I 286 Belagerung von Stuttgart

I 3 10 Reichskrieg Hein-

richs VII. gegen Eberhard.

Diesen rettet Heinrichs Tod

Eberhard der Erlauchte ver-

I325-I344 Graf Ulrich III. Er erwirbt die Herrschaft Teck und beginnt im größeren Stil durch Kauf sein Gebiet zu erweitern ( 1342 die Stadt Tübingen mit dem Schönbuch). 1336 endgültige Erwerbung Markgröningens mit der Reichssturmfahne

1344-1362 Graf Ulrich IV. und 1344-1392 Graf Eberhard der Greiner

I 377 Niederlage vor Reutlingen durch Schuld seines Sohnes Ulrich

r 378 Belagerung von Stuttgart

I 388 Schlacht bei Döffingen; Sieg Eberhards über die Städte, sein Sohn Ulrich fällt

1392-1417 Graf Eberhard der Milde legt das Chorherrnstift Beutelsbach nach Stuttgart. Starker Ausbau Stuttgarts

I 274/75 in Augsburg entsteht der Schwabenspiegel, eine Aufzeichnung des Gewohnheitsrechts

Aufblühen der am Fernhandel beteiligten Städte

I 347 Baubeginn der Stuttgarter Stiftskirche

I 377 Baubeginn des Ulmer Münsters

Gotischer Baustil

Beginnender Niedergang der politischen Macht der Städte. Wirtschaftliche und kulturelle Blüte der schwäbischen Reichsstädte 
EUROPÄISCHE

GESCHICHTE

14 10-1 437 Kaiser Sigis-

mund (aus dem Haus Lu-

xemburg)

1414-1418 Konzil von Kon$\operatorname{stan} z$

seit 1438 Kaiser aus dem Hause Habsburg um 1450 Erfindung des Buchdrucks
1493-1 5 I9 Kaiser Maximilian I.
POLITISCHE

ENTWICKLUNG IN

WÜRTTEMBERG

1417-1419 Graf Eberhard

der Jüngere

Nach Eberhards Tod regiert seine Gemahlin Henriette von Mömpelgard für ihre unmündigen Söhne:

1419/26-1450

Graf Ludwig I.

1419/33-1480

Graf Ulrich V., der Vielge-

liebte

1442 Teilung des Landes in

den Stuttgarter (Ulrich)

und den Uracher Landesteil

(Ludwig)

1462 läßt sich Ulrich V. zum schweren Nachteil sei-

nes Landes in die "Pfälzer

Fehde" verwickeln

1450 Vormundschaftsregierung für Ludwigs unmündige Söhne:

1450-1457 Graf Ludwig II. 1450-1496 Graf EberhardV., später Eberhard im Bart 1459 Eberhard für volljährig erklärt

1482 Wiedervereinigung der beiden Landesteile durch den Münsinger Vertrag; der gleichnamige Vetter Eberhards im Bart verzichtet auf die Regierung des Stuttgarter Landesteils, er soll Nachfolger Eberhards im Bart werden, der keine legitimen Kinder hat

1492 Eßlinger Vertrag, Sicherung der Unteilbarkeit des Landes
GEISTIGES LEBEN IN WÜRTTEMBERG
Der künstlerisch begabte Ulrich läßt den Bau der StuttgarterStiftskirche fortsetzen. Beginn des Baues der Leonhards- und Hospitalkirche in Stuttgart. Baumeister:

Hänslin Jörg, Gründer der Stuttgarter Bauhütte und sein Sohn Aberlin Jörg

1477 Eberhard im Bart gründet unter dem Einfluß seiner Mutter Mechthild von der Pfalz und seines Lehrers und Ratgebers Vergenhans (Nauclerus) die Universität Tübingen

Verlegung des Sindelfinger Chorherrnstiftes nach Tübingen 
EUROPÄISCHE

GESCHICHTE

I 495 Reichstag zu Worms (ewiger Landfriede, Reichskammergericht)

allmähliche Einführung des römischen Rechtes

das Rittertum verliert seine militärische Bedeutung

1517 Beginn der Reformation durch Martin Luther

1519-1556 Kaiser Karl V., sein Bruder König Ferdinand I., 1 556-1564 Kaiser

1524-1525 Bauernkrieg

1546-1547 Schmalkaldischer Krieg

\author{
POLITISCHE \\ ENTWICKLUNG IN \\ WÜRTTEMBERG
}

I 495 Eberhard im Bart zum Herzog erhoben

1496-I 498 Herzog Eberhard II.

1498 Eberhard II. wird durch die Landstände abgesetzt

Regentschaft der Stände für Eberhards Neffen:

I 498-1550 Herzog Ulrich, Sohn des geisteskranken Grafen Heinrich

1503 Ulrich wird vom Kaiser für volljährig erklärt

1504 bedeutender Landgewinn Ulrichs im Krieg zwischen Kurpfalz und Bayern um die Erbschaft Herzogs Georg des Reichen von Bayern-Landshut

I5I4 Aufruhr des "Armen Konrad " und Tübinger Vertrag, der das Grundgesetz Altwürttembergs wird 1515 Ulrich ermordet seinen Stallmeister Hans von Hutten

1519 Ulrich annektiert die Reichsstadt Reutlingen I519 Vertreibung Ulrichs aus seinem Herzogtum durch den Schwäbischen Bund 1520-1534 Württemberg Besitz des Hauses Habsburg

I 534 Ulrich erobert Württemberg mit Hilfe Philipps von Hessen zurück. Vertrag von Kaaden:

Ulrich erhält sein Land zurück, aber nur als österreichisches Lehen

1547 Heilbronner Unterwerfung;
GEISTIGES LEBEN

IN WÜRTTEMBERG

1425-1510 JohannesVergenhans, weit über Württemberg hinaus bekannter Gelehrter, erster Rektor und später Kanzler der Universität Tübingen

I 495 Landesordnung (erste einheitliche Gesetzgebung für das ganze Land)

1455-1522 Johannes Reuchlin, galt als Haupt der deutschen Humanisten, Rat der Herzöge Eberhard im Bart und Ulrich, Professor des Griech. und Hebr. an der Universität Tübingen ( 152 I bis 1522 )

Melanchthon in Tübingen (1512-1518), Aufblühen humanistischer Schulen in Schwaben

Einführung der Reformation im Herzogtum Württemberg. Reformation der Universität Tübingen gegen den Widerstand vieler Professoren durch Blarer und Brenz, Berufung eines neuen akademischenLehrkörpers, Umwandlung der Universität aus einer kirchlichen in eine landesherrlicheAnstalt. 
EUROPÄISCHE

GESCHICHTE

1555 Augsburger Religionsfriede

1564-1576 Kaiser Maximilian II.

I 576-16 I 2 Kaiser Rudolf II., Zuspitzung der konfessionellen Gegensätze
GEISTIGES LEBEN

IN WÜRTTEMBERG

ENTWICKLUNG IN WÜRTTEMBERG

Ulrich soll sich vor seinem Lehnsherrn König Ferdinand I. rechtfertigen

Neuordnung des Kirchenwesens durch Erhard Schnepf und Ambrosius Blarer

1550-1568 Herzog Christoph, unter den europäischen Fürsten hochangesehen als Staatsmann und Diplomat

1552 Landesordnung

1553 Einigung mit Ferdinand I., der Ulrich als treubrüchigen Lehnsmann angeklagt hatte und das Herzogtum wieder an sich ziehen wollte

1552 Feststellung der Religion des Landes in der Confessio Virtembergica

1555 Verkündung des württembergischen Landrechtes 1556 Umwandlung der Mannsklösterin Klosterschulen zur Vorbildung des geistlichen Nachwuchses. Errichtung des Tübinger "Stifts" 1559 Große Kirchenordnung

Unter Herzog Christoph Bau des Stuttgarter Alten Schlosses durch Aberlin Tretsch

Jakob Heerbrand ( 1521 bis 1600), Professor der Theologie und Kanzler der Universität Tübingen, Vertreter Württembergs beim Konzil von Trient

1568-1593 Herzog Ludwig Jakob Andreä (1528-1590), Professor der Theologie und Kanzler der Universität Tübingen, bedeutender Vertreter der lutherischen Orthodoxie, Verfasser der Konkordienformel

Nikodemus Frischlin (1547 bis 1590 ), bedeutender humanistischer Poet von starker satirischer Begabung

Unter dem kunstsinnigen Herzog Ludwig erbaut Georg Beer in Stuttgart das Lusthaus, Simon Schlör ge- 
EUROPÄISCHE

GESCHICHTE

16 8 Ausbruch des Dreißig-

jährigen Krieges

1619-1637 Kaiser Ferdi-

nand II.

1620 Schlacht am Weißen

Berge bei Prag

1629 Restitutionsedikt

1630 Eingreifen König Gu-

stav Adolfs von Schweden

zugunsten der Protestanten
1593-1608 Herzog Friedrich I.

Da Ludwig kinderlos stirbt folgt ihm der Sohn von Herzog Ulrichs jüngerem Halbbruder Georg

Politischer und wirtschaftlicher Absolutismus. Sein Kanzler ist Matthäus Enzlin

\section{Prager Vertrag}

(Ablösung des Lehnsverhältnisses zu Österreich)

I607 Änderung des Tübinger Vertrags, Zurückdrängung der Macht der Stände

1608-1628 Herzog Johann Friedrich

Die Landstände gewinnen ihre Macht zurück. Enzlin wird enthauptet $\left(16 \mathrm{I}_{3}\right)$

I628/33-1674 Herzog Eberhard III.

Für den unmündigen Eberhard führen zwei Brüder Johann Friedrichs als Her-
GEISTIGES LEBEN

IN WÜRTTEMBERG

staltet die Grafenstandbilder in der Stiftskirche zu Stuttgart

Martin Crusius (1526-1607), Professor an der Tübinger Artistenfakultät schreibt "Schwäbische Annalen"

1592 Errichtung des bereits von Christoph geplanten Collegium illustre zu Tübingen als Erziehungsanstalt für weltliche Beamte

I 596 Umwandlung des Collegium illustre in eine Fürsten- und Adelsschule

Friedrich fördert sehr die Künste:

Sein Baumeister Heinrich Schickhardt (1558-1634)

legt die Stadt Freudenstadt an (1599)

Schickhardt errichtet im Stil der Renaissance in Stuttgart den "Neuen Bau"

Johannes Kepler (1571 bis 1630), im Tübinger Stift I 589, Hofastronom Rudolf II.

Johann Valentin Andreä (1584-1654), Vorläufer des Pietismus und vielseitiger Gelehrter

Der Dreißigjährige Krieg zieht auch die Universität Tübingen in Mitleidenschaft; zahlreiche Lehrstühle bleiben unbesetzt, Studenten gibt es nur noch wenige

Die frühbarocke Dichtung ist in Württemberg durch 
EUROPÄISCHE

GESCHICHTE

I634 Sieg der Kaiserlichen über die Schweden bei Nördlingen

I635 Frankreich greift unter der Führung Richelieus $(1585-1642)$ in den Krieg ein

I648 Friede zu Münster und Osnabrück

1643-1715 Ludwig XIV. von Frankreich

\section{POLITISCHE}

ENTWICKLUNG IN WÜRTTEMBERG

zöge-Administratoren die Regierung:

1628-163 I Herzog Ludwig Friedrich von WürttembergMömpelgard

1631-1633 Herzog Julius Friedrich von WürttembergWeiltingen

I633 Eberhard für mündig erklärt

I $634-1638$ Eberhard desLandes vertrieben

Eberhard hat bedeutende

Männer als Kanzler und Räte:

Jakob Löffler (1583-1638) Andreas Burkard

(gest. I648)

Konrad Varnbüler (I 595 bis r657)

1658-1 705 Kaiser Leopold I. Türkenkriege
I70I-I714 Spanischer Erbfolgekrieg
GEISTIGES LEBEN

IN WÜRTTEMBERG

Georg Rudolf Weckherlin vertreten, gestorben 1653 in London

I642 Einführung der Kirchenkonvente nach reformiertem Vorbild zur Ausübung der Kirchenzucht

I 649 Einführung des grundsätzlich zwangsmäßigen Volksschulbesuchs
1674-1677 Herzog Wilhelm Ludwig

I677/93-1 733 Herzog Eberhard Ludwig;

während seiner Minderjährigkeit führen die Regierung Herzog-Administrator Friedrich Karl von Württemberg-Winnenthal, ein Bruder Wilhelm Ludwigs, als Vormünderin die Herzoginwitwe Magdalene Sibylle von Hessen-Darmstadt

I693 Eberhard Ludwig volljährig
I699 Aufnahme von 3000 aus Piemont vertriebenen Waldensern unter ihrem Führer Henri Arnaud

Seit I704 läßt der Herzog von Johann Friedrich Nette, Giuseppe Frisoni und Paolo Retti Schloß und Stadt Ludwigsburg erbauen (Barock)

Kirchlicher Barock in Oberschwaben (Neuwürttemberg)

\section{Kirchlicher Barock}

Steinhausen (Pfarrkirche 1727-1733 von Domenicus Zimmermann und seinem Bruder Johann)

Siessen (Klosterkirche 1726-I 733 von Domenicus Zimmermann und seinem Bruder Johann; Klostergebäude I 7 I I von Christian Thumb) 
Schussenried (Bibliothekssaal des Klosters $1754-176$ I nach Plänen von Domenicus Zimmermann von Jakob Emele; Barockausstattung der Kirche I 7 10-1 746)

Obermarchthal (Klosterkirche I686-170I von Michael Thumb, Christian Thumb und Franz Beer; Klostergebäude 1686-1702 und der NO-Flügel $1747-1753$ von G. G. Bagnato)

Weingarten (Klosterkirche I715-1724 nach Plänen Franz Moosbruggers von Franz Beer, Andreas Schredk, Christian Thumb und D. G. Frisoni)

Zwiefalten (Klosterkirche 1739-1765 von Joseph und Martin Schneider und J. M. Fischer; Klostergebäude 1668-1690 von Thommaso Comacia, Michael Thumb, Heinrich Mayer und Franz Beer)

Weissenau (Langhaus der Klosterkirche 1717-1724 von Franz Beer; Klostergebäude I 708-1 7 I 7 von demselben)

Friedrichshafen (Schloßkirche - ehemalige Benediktinerprioratskirche Hofen 1695-1701 von Christian Thumb; ehemalige Klostergebäude 1654-166I von Franz Beer und 1697-1701 von Christian Thumb)

Ehingen (Konviktskirche 1712-1719 wahrscheinlich von Franz Beer; Kollegiengebäude 1698-1706 von Franz Beer)

Wiblingen (Klosterkirche $1772-1781$ von Johann Georg Specht nach dem Vorbild der Kirche in St. Gallen von Peter Thumb; Klostergebäude 1714-1762 von Christian Wiedemann und Johann Michael Fischer)

Beuron (Klosterkirche $1732-1738$ von Matth. Scharpf)

Erbach (Pfarrkirche 1767-1769 von Franz Kleinhans)

Ochsenhausen (Klosterkirche I 725-1732 Barockfassade von Christian Wiedemann, barocke Ausstattung des Inneren der Kirche)

Wolfegg (Schloßkirche 1733-1742 von Johann Georg Fischer)

Rot a.d. Rot (Klosterkirche 1777-1786; Klostergebäude von 1682-1702)

Deggingen (Pfarrkirche 1700; Wallfahrtskirche 1716-1718)

EUROP ̈̈ISCHE GESCHICHTE

I 7 I 1 -I 740 Kaiser Karl VI.

1740-1 780 Maria Theresia, Königin von Böhmen und Ungarn, Erzherzogin von Osterreich

I 745-I 765 Kaiser Franz I.

1 740-1 786 Friedrich der

Große, König von Preußen

1756-1763 Siebenjähriger

Krieg
POLITISCHE

ENTWICKLUNG IN WÜRTTEMBERG
GEISTIGES LEBEN

IN WÜRTTEMBERG

Bedeutende Männer der

Zeit:

Georg Bernhard Bilfinger (1693-1750), Philosoph aus der Schule von Leibniz und Wolff, württembergischer Konsistorialpräsident

Johannes Osiander ( 1657 bis I724), Direktor des Konsistoriums und wirkl. Geheimer Rat, häufig als geschätzter Diplomat im Ausland tätig 
EUROPÄISCHE GESCHICHTE

1789 Beginn der französischen Revolution

I792-1805 Koalitionskriege gegen Frankreich
POLITISCHE

ENTWICKLUNG IN

WÜRTTEMBERG

1733-1737 Herzog Karl

Alexander,

Sohn des Herzog-Admini-

strators Friedrich Karl von

Württemberg-Winnenthal

1737/44-1793 Herzog Karl Eugen

Bis zur Volljährigkeit Karl Eugens 1744 Vormundschaftsregierung durch: 1737-1738 Karl Rudolf von Württemberg-Neuenstadt 1738-1744 Friedrich Karl von Württemberg-Oels
1793-1795 Herzog Ludwig Eugen, Bruder Karl Eugens

1795-1 797 Herzog Friedrich Eugen, Bruder Karl Eugens
GEISTIGES LEBEN

IN WÜRTTEMBERG

Christoph Matthäus Pfaff (1686-1760), Professor der Theologie und Kanzler der Universität Tübingen, bemüht sich um die Vereinigung von Lutheranern und Reformierten

Johann Albrecht Bengel (1687-1752), Prälat und Konsistorialrat, man kann ihn den "Vater des schwäbischen Pietismus" nennen

Johann Jakob (v.) Moser (I70I-I 785), württ. Landschaftskonsulent, man nennt ihn "Vater des deutschen Staatsrechts"

Friedrich Christoph Oetinger (I702-1782), Schüler Bengels, Mystiker und Theosoph ("Magus des Südens"), zuletzt Prälat in Murrhardt

Christian Friedrich Daniel Schubart (1739-1792), Publizist, vertritt als Dichter in Württemberg den Sturm und Drang

Philipp Matthäus Hahn (1739-1790), württembergischer Pfarrer, ein mechanisches Genie

I775 Verlegung der Karlsschule von der Solitude nach Stuttgart

I78I von Kaiser Joseph II. unter dem Namen Hohe Karlsschule zur Universität erhoben

1793 von Herzog Ludwig Eugen wieder aufgehoben

Friedrich Schiller (I 759 bis I 805) 
EUROPÄISCHE

GESCHICHTE

I 798 Napoleon, erster Konsul der Französischen Republik

I 803 Reichsdeputationshauptschluß

I 804-1814 Napoleon I., Kaiser der Franzosen

I 806 Ende des alten Deutschen Reiches

I813-1815 Befreiungskriege

I 8 14-I 8 I 5 Wiener Kongreß; Errichtung des Deutschen Bundes
POLITISCHE

ENTWICKLUNG IN WÜRTTEMBERG

I796 Sonderfriede mit der Französischen Republik

I 797-I 8 I 6 Friedrich II.

(Herzog bis 1803 )

r799 Teilnahme am Krieg gegen Frankreich

I 802 Friede mit Frankreich

I 803 Friedrich wird Kurfürst; Neuwürttemberg

I 805 Anschluß an Napoleon

I 806 Württemberg wird Königreich mit voller Souveränität

I 806 Beitritt zum Rheinbund

I 8 I 3 nach der Schlacht bei Leipzig Anschluß an die Verbündeten

I 8 I 5 Beitritt zum Deutschen Bund, nicht ohne Widerstreben Friedrichs I.

I 815 Kampf um die Neugestaltung der Verfassung
GEISTIGES LEBEN

IN WÜRTTEMBERG

Johann Heinrich Dannecker (1758-1838), bedeutender Bildhauer der Zeit

Karl Friedrich (v.) Reinhard (176I-1837), seit r 793/95 französischer $\mathrm{Di}$ plomat, I798 Außenminister der französischen Republik

Georg Wilhelm Friedrich Hegel (1770-183r)

Friedrich Wilhelm Schelling (I 775-r 854)

Friedrich Hölderlin ( 1770 bis 1843 )

Ludwig Uhland ( 1787 bis I862), "Dichter, Gelehrter und Politiker in einer Person "

Friedrich List (1789-1846), Nationalökonom, kämpft für ein einheitliches deutsches Wirtschaftsgebiet

1 $817 / 18$ Verlegung $\operatorname{der} 18$ I 2 errichteten katholisch-theologischen Universität Ellwangen als katholisch-theologische Fakultät nach Tübingen

I 828 Errichtung des Bistums Rottenburg 
EUROPÄISCHE

GESCHICHTE

I 848-I 849 revolutionäre

Unruhen in Deutschland

I 848 Deutsche Nationalversammlung in Frankfurt
1866 Kampf zwischen Osterreich und Preußen um die Vorherrschaft in Deutschland

1870-1 87 I Deutsch-Französischer Krieg
POLITISCHE

ENTWICKLUNG IN WÜRTTEMBERG

GEISTIGES LEBEN

IN WÜRTTEMBERG

I 8 I 6-I 864 König Wilhelm I.

I 819 Verkündung der Verfassung; Württemberg eine konstitutionelle Monarchie

I 849 das "Rumpfparlament" in Stuttgart (am 18 . Juni von der württembergischen Regierung aufgelöst)

1864-1891 König Karl

I866 Teilnahme am Krieg gegen Preußen

I 870 Teilnahme am Krieg gegen Frankreich an der Seite des Norddeutschen Bundes
Als Dichter und Schriftsteller sind für diese Zeit zu nennen:

Wilhelm Hauff (1 802-1 827)

Wilhelm Waiblinger (I 804 bis 1830 )

Eduard Mörike ( 1804 bis 1875)

Justinus Kerner ( $\mathrm{I} 786$ bis 1862), besonders bekannt durch seine Schriftstellerei auf okkultem Gebiet

Friedrich Theodor Vischer (1807-1887), Professor der deutschen Literatur und Ästhetik, Schriftsteller

Ferdinand Christian Baur (1792-1860), Professor der Kirchen- und Dogmengeschichte, Begründer der historisch-kritischen Schule Tübingens

David Friedrich Strauß (1 808-1874), Links-Hegelianer, Begründer der "Leben Jesu"-Forschung

Johann Adam Möhler ( 796 bis 1838 ), Professor der katholischen Theologie, Begründer der "Tübinger Schule»

Karl Joseph Hefele (1809 bis 1893), Kirchenhistoriker, 1869 Bischof von Rottenburg

Robert Mayer (1814-I878) Arzt und Physiker, Entdekker des Gesetzes von der Erhaltung der Energie 


\section{ENTWICKLUNG IN WÜRTTEMBERG}

I87 I Zusammenschluß der süddeutschen Staaten mit dem Norddeutschen Bund zum Deutschen Reich
1914-1918 erster Weltkrieg

1918 Sturz der Monarchie im Reich und in den Bundesstaaten
I 87 I Das Königreich Württemberg wird ein Bundesstaat des Deutschen Reiches

Wenn auch Württemberg als Staat erhalten blieb, so endet damit doch eine selbständige württembergische Geschichte

I89 I-1918 KönigWilhelm II. 1906 Verfassungsrevision

19 8 Abdankung König Wilhelms II.

I919 neue republikanische Verfassung
Erzäbler:

Hermann Kurz (1813 bis 1873) und der Ingenieur Max Eyth (1836-1906)

Wissenschaft -

Rechtswissenschaft:

Karl Georg (v.) Wächter

(I797-I 880)

Staatswissenschaft:

Robert (v.) Mohl (1797 bis I875)

Historiker:

Ludwig Friedrich Heyd

(1792-1843) und

Christoph Friedrich Stählin (1805-1873)

protestantische Theologen:

Tobias Beck (1804-1878)

und Karl Weizsäcker (1822 bis 1899 )

Technik:

Gottlieb Daimler (1834 bis 1900)

Graf Ferdinand v. Zeppelin (1838-1917)

Robert Bosch (1861-1942)

I 876 Erhebung des Polytechnikums in Stuttgart zur

Technischen Hochschule

1909 Revision des Volksschulgesetzes von 1836 (Abschaffung der Schulaufsicht durch Geistliche)

\section{Malerei:}

Graf Leopold v. Kalckreuth (1855-1928), lehrte an der Stuttgarter Kunstakademie

Adolf Hölzel (1853-1934) Willi Baumeister (1 899 bis 1955)

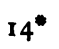


EUROPÄISCHE

GESCHICHTE

I919 neue Reichsverfassung, das Reich eine Republik

1933 Ubernahme der Macht im Reich und in den Ländern durch die NSDAP unter Adolf Hitler

1934 die Hoheitsrechte der Länder gehen auf das Reich über

1939-1945 zweiterWeltkrieg

1945 bedingungslose Kapitulation des "Großdeutschen Reiches"; Aufteilung Deutschlands in vier Besatzungszonen

1949 Errichtung der Bundesrepublik Deutschland (umfaßt die drei westlichen Besatzungszonen)
POLITISCHE

ENTWICKLUNG IN

WÜRTTEMBERG

Staatspräsidenten:

1919-1920 Wilhelm Blos (1849-1927)

1920-1924 Johannes Hieber (1862-1951)

1924-1928 Wilhelm Bazille (1874-1934)

1928-1933 Eugen Bolz (1 881-1944)

Aufhebung des Amtes des Staatspräsidenten, Reichsstatthalter 1933-1945 Wilhelm Murr (1888-1945)

1945 Teilung Württembergs in eine nördliche und eine südliche Hälfte; Bildung der Länder Württemberg-Baden und Württemberg-Hohenzollern

1952 Vereinigung der Länder Württemberg-Baden, Württemberg-Hohenzollern mit dem Land Baden (südliche Hälfte) zum Land Baden-Württemberg
GEISTIGES LEBEN

IN WÜRTTEMBERG

Arcbitektur - Häupter der Stuttgarter Architektenschule:

Theodor Fischer ( 1862 bis 1938)

Paul Bonatz (1877-1955)

Paul Schmitthenner (geb. I 884)

\section{Literatur:}

Isolde Kurz (1853-1944)

Ludwig Findkh (geb. 1876 )

Hermann Hesse (geb. 1877)

August Lämmle (geb. 1876)

1924 Trennung von Kirche und Staat

evangelische Theologie:

Adolf Schlatter (1 852 bis 1938)

Karl Heim (1884-1958)

Gescbichtswissenschaft:

Johannes Haller (1865 bis 1947)

alle drei Professoren der Universität Tübingen

1934 Kirchenkampf: evang. Kirche: Theophil

Wurm (1868-1953), 1929 Kirchenpräsident, 1933 bis 1948 Landesbischof, 1945 bis 1948 Vorsitzender des Rates der evang. Kirche in Deutschland

kath. Kirche: Johannes B. Sproll (1870-1949), Bischof von Rottenburg 